\title{
12 NS-Ratgeberliteratur: Anleitungen zum „rassenhygienischen“ Erfolgstypus zwischen arbeitspsychologischem und metaphysischem Glückswissen
}

\section{1 „Schicksalsmächte des Erfolges?“: Autosuggestive Selbsterziehung zum „Erfolgstyp“ im Glücksratgeber von C.H. Huter}

„Schicksalsmächte des Erfolges?“ - „Gehören Sie zu einem Erfolgstyp?“1 Diese suggestiven Fragen wirft das Titelblatt (Abb. 6) des Erfolgs- und Glücksratgebers „Schicksalsmächte des Erfolges?“ des Astrologen und Esoterikers Carl Heinrich Huter (1898-1974) auf. Die Antwort darauf lautet: Das „Glück“ der Einzelperson hänge von der „Gegenseitigkeit der Leistungen, der Handlungen und der Taten ab“, so C.H. Huter. ${ }^{2}$ Im Kapitel zur „Persönlichkeitskultur“ bringt der Autor dieses Abhängigkeitsverhältnis auf den Punkt: Um das „Ich zu stärken“ sollte der „Willensmoment“ nicht von „egoistische[m] Streben[...]“ bestimmt sein. Eine „hohe Persönlichkeit“ könne nur der „höchste Diener seiner Gemeinschaft“ sein. ${ }^{3}$ Das individuelle Arbeitsglück stehe, laut C.H. Huter, in Abhängigkeit zum Dienst an der Gemeinschaft.

Welche Wissensbestände rezipierte C.H. Huter zur Beantwortung der Fragen über „Glück“, „Erfolg“ und „Lebensführung“ und wie wurden diese im Kontext der psychopolitischen NS-Arbeitsmoral umgedeutet? Welche wissenshistorischen Referenzpunkte lassen sich aus den Ratgeberpraktiken erschließen und welche Erkenntnisse können für die Analyse einer transnationalen Politik der ,Glückskulturen' gewonnen werden? Diese Fragen werden im vorliegenden Kapitel zu beantworten versucht. Doch zunächst: Wer war der Autor dieses Ratgebers?

Der Ratgeberautor war der Sohn des berühmten Physiognomikers Carl Huter (1861-1912). ${ }^{4}$ Physiognomik nach Huter sei, laut Fritz Aerni ${ }^{5}$, die „Deutung des

1 Titelblatt, vgl. Huter 1940 und Abb. 6.

2 Vgl. Huter 1940, 8.

3 Vgl. ebd., 14.

4 Carl Huter (1861-1912) wurde 1861 in Heinde bei Hildesheim in Niedersachsen geboren und starb 1912 in Dresden. Nach dem Abschluss seiner Lehre als Dekorations- und Porträtmaler in Hildesheim zog es ihn nach Berlin, Leipzig und Dresden. Dort bildete er sich als Autodidakt interdisziplinär in Kunstgeschichte, Philosophie, Kulturanthropologie, Medizin, Naturwissenschaften und Psychologie weiter. An einem schweren, als unheilbar diagnostizierten Halsleiden

Ә OpenAccess. (c) 2021 Isabelle Haffter, publiziert von De Gruyter. (cc))BY-NC-ND Dieses Werk ist lizenziert unter einer Creative Commons Namensnennung - Nicht kommerziell - Keine Bearbeitung 4.0 International Lizenz. https://doi.org/10.1515/9783110661439-012 
Erscheinungsbildes, der Physiognomie eines Menschen.“6 Das psycho-physiognomische Analysemodell aus Huters Hauptwerk „Menschenkenntnis“7 (19041906) erhebt den wissenschaftlichen Anspruch, aus dem individuellen, physischen, sinnlich wahrnehmbaren Erscheinungsbild eines Menschen auf das Wesen, das heißt, auf 1. die Formen individuell wirkender psycho-physiologischer „Kräfte“, 2. auf den „Charakter“ und 3. auf die „seelischen Eigenarten“ schließen zu können. ${ }^{8}$ Nach dem Tod seines Vaters verdiente C.H. Huter mit dem Vertrieb der Huterschen Lehrbücher und seinen eigenen Schriften ein Vermögen. ${ }^{9}$ Am 1. Mai 1933 trat der Esoteriker der NSDAP bei. ${ }^{10}$

Der Ratgeberautor C.H. Huter kann exemplarisch für die transnationale Rezeption, Transformation und Umdeutung von arbeitspsychologischem Glückswissen von der Zeit des Wilhelminischen Kaiserreichs über die Weimarer Republik bis in die NS-Zeit stehen. C.H. Huters enge Verbindung zum NS-Regime wird bei

erkrankt, wandte er sich auf der Suche nach einer Selbsttherapie den physiognomischen Lehren zu. Im Laufe seiner Forschungstätigkeit weitete er die Lehransätze auf der Grundlage der damaligen psychologischen Erkenntnisse zur sogenannten Psycho-Physiognomik aus. Vgl. Aerni 2017.

5 Dem aktuellen Forschungsstand zufolge existieren kaum wissenschaftshistorische Auseinandersetzungen mit Carl Huters Lehren. Aus diesem Grund müssen die Literaturverweise zu C. Huters Leben und Werk, die sich im Wesentlichen auf die Publikationen des Carl-Huter-Instituts beziehen, kritisch reflektiert werden. Zur Kritik an der Psycho-Physiognomie und deren aktueller Rezeption im Personalmanagement von Betrieben vgl. Kanning 2002, Kanning 2007, Kanning 2010. Eine Publikationsauswahl des Huter-Instituts: Fleischhacker 1983, Aerni 2012b, Aerni 2017, Huter 2020.

6 Vgl. Aerni 2001, 17.

7 Vgl. Huter 2020. Zum Zeitpunkt der Niederschrift dieses Buches ist die Neuauflage noch nicht erschienen. Verweise auf das Werk „Menschenkenntnis“ beziehen sich aufgrund der Rezeptionsdebatte der Hutscherschen Lehre betreffend rassistischer Inhalte auf einen Vergleich der Publikationen des gleichnamigen Werks im Huter-Verlag, 4. Aufl.von 1991, vgl. Huter 1991, und der Ausgabe des Kalos-Verlags von 1992, hg.v. Fritz Aerni, vgl. Huter 1992.

8 Zur Rezension von C. Huters und C.H. Huters Schriften im Nationalsozialismus bis zu deren Verbot 1937 bzw. 1941, vgl. Aerni 2012a. Aufschlussreich sind darin die beschriebenen „Anbiederungsversuche“ des C. Huter-Verlegers Amadeus Kupfer (1879-1952) mittels „rassenhygienischer“ Publikationen in der Schweizer Zeitschrift „Der Menschenkenner und die neue Zeit nach Carl Huters Psycho-Physiognomik“ zwischen 1932 und 1934 an das NS-Regime. Vgl. u.a. Aerni 2012a, 626-636.

$9 \mathrm{Zu}$ den Schriften C.H. Huters vgl. u. a. Huter 1927.

10 Vgl. Korrespondenz mit Fritz Aerni, Huter-Institut, Zürich, 02.10.2019. Vgl. Aerni 2012a, 654. Zum ideologischen Streit zwischen C.H. Huter in Dresden/Stuttgart, der Verlagsfamilie Kupfer in Arlesheim b. Basel und den unterschiedlichen Verlegern in Zürich (z. B. Walter Alispach, KaliosVerlag) über die verlegerischen Aktivitäten der verschiedenen Huter-Bewegungen während der NS-Zeit, über NS-Sympathien einzelner Schweizer Huter-Anhänger^innen und Deutungshoheiten der Huterschen Lehre während und nach der NS-Zeit, vgl. Aerni 2012a, 556f. 


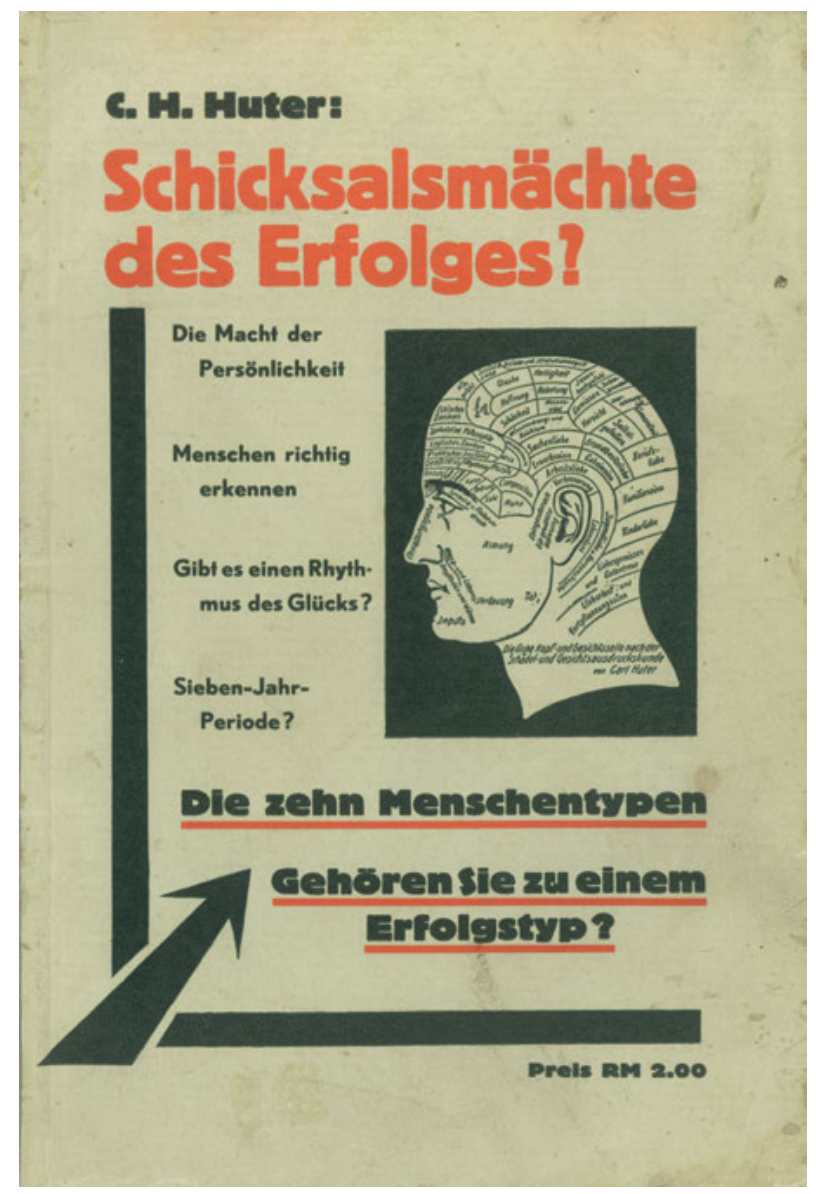

Abb. 6: NS-Ratgeber von C.H. Huter „Schicksalsmächte des Erfolges?“, 1940

der wissenshistorischen Analyse seines Ratgebers offenkundig. Die „Grundsätze der NSV.-Arbeit“, welche auf mehreren Seiten des Ratgebers abgedruckt wurden, lassen eine Zusammenarbeit zwischen dem C.H. Huter Verlag und der „Nationalsozialistischen Volkswohlfahrt (NSV)“ vermuten. ${ }^{11}$

11 Ein Beispiel: „Grundsatz der NSV.-Arbeit: Jeder einzelne Volksgenosse muß Mitkämpfer sein, stark und opferbereit“. Vgl. Huter 1940, 42. 
Der Autor griff in seinem Ratgeber auf Wissensbestände der Psycho-Physiognomik-Lehre seines Vaters, der arbeitswissenschaftlichen Psychotechnik ${ }^{12}$ sowie der „,ölkischen“ Arbeitsmetaphysik zurück und deutete diese als praktische Anleitungen für eine erfolgreiche und „glückliche“ Lebensführung im Kontext der „rassenhygienischen“ NS-Arbeitsmoral und Kriegspropaganda um. Wie ging er dabei vor?

Den „Sinn des Lebens“ sah C.H. Huter in der teleologischen „Höherentwicklung der Kultur“, die von einem energetischen „Kampf“ und einer menschlichen „Schaffenskraft“ geprägt sei. ${ }^{13}$ Von dieser wissenshistorischen Prämisse ausgehend, verortet sich der Glücksratgeber innerhalb unterschiedlicher esoterischer und post-nietzscheanischer „Willenskraft“-Diskurse, ähnlich Gieses arbeitspsychologischer Kulturphilosophie und energetischer „Arbeitsmetaphysik“ (s. Kap. 11.1, 12.3).

Anders als in Baerwalds Nietzsche-Interpretation der „amor fati“ besäße der Mensch nach C.H. Huter einen Handlungsspielraum als „Mitwirkend[er] auf der Lebensbühne““. ${ }^{14}$ Dieser ,Theater'-Metapher liegt eine leistungsorientierte, selbsterzieherische und autosuggestive Prämisse zugrunde. Ähnlich einer Schauspieler^in, die auf der Bühne keine Autonomie besäße, sondern in Abhängigkeit zur Regieanweisung und Dramaturgie ihre Rolle bestmöglich ausführte, müssten die Leser`innen den vom Ratgeberautor C.H. Huter normativ festgelegten Spielregeln folgen, um „Glück“ und Erfolg zu erlangen:

„Die Geheimnisse erfolgreicher Menschen liegt [sic] nicht allein in dem persönlichen Glück, sondern vielmehr in der Erkenntnis der genannten Erfolgsgesetze, die in verschiedenen Lebenslagen auswertbar sind. Richtige Nutzbarmachung unserer Persönlichkeit muß einen Aufstieg im Leben bewirken.“15

Nur dem Menschen sei Erfolg sicher, der „eine sinnvolle Lebensplanung schafft und die eigene Persönlichkeit durch Selbsterziehung und Willensschulung wachsen läßt“", lautet Huters Erfolgsgesetz. ${ }^{16}$ Dieses Beispiel aus der NS-Zeit zeigt: Auch in C.H Huters Glücksratgeber von 1940 bildet sich die Zirkulation von Wissen über eine leistungsorientierte „Selbsterziehung“ und autosuggestive

12 Huter zitiert beispielsweise einen Aufsatz aus dem Sammelband „Psychotechnik. Angewandte Psychologie“, der 1935 vom Arbeitspsychologen Hanns Spreng herausgegeben und im Max Niehaus Verlag in Zürich und Leipzig publiziert wurde. Vgl. Huter 1940, 46. Vgl. Spreng 1935.

13 Vgl. Huter 1940, 1.

14 Vgl. ebd., 1.

15 Ebd., 4.

16 Vgl. ebd., 1. 
„Willensschulung“ aus der Wilhelminischen Ratgeberliteratur und der Weimarer Republik in den zentralen Begriffen der praktischen Anleitung ab.

Als höchstes Ziel des menschlichen Lebens definiert C.H. Huter das Erreichen der „Wunschbahn des Lebenserfolges“, so sein zentraler Lehrsatz. ${ }^{17}$ Was versteht C.H. Huter unter „Lebenserfolg“?

„[...] in der Hauptsache [geht es] um die Entwicklung einer leistungsfähigen Persönlichkeit, die befähigt ist, materielle und ideelle Werte zu schaffen, um diese wiederum für die Gemeinschaft einzusetzen. Denn allein dies sichert einen dauernden Erfolg, der von einer segenreichen Grundlage gekrönt ist.“18

Wie schon in Gieses Ratgeber über eine „eugenische“ Wissenskultur einer energetischen und biologistischen „Körperseelenmeisterung“ wird auch bei C.H. Huter die „Entwicklung einer leistungsfähigen Persönlichkeit“ und die Schaffung „materieller und ideeller Werte“ in den arbeits- und kulturideologisch überhöhten Dienst der Gemeinschaft (oder mit Ley der „Volksgemeinschaft“) gestellt. Ein individuelles Streben nach autonomer Selbstverwirklichung wird der Einzelperson im Sinne der NS-Arbeitsideologie und dessen Gesellschaftsutopie einer imaginierten Einheit aus Staat und „Volksgemeinschaft“ abgesprochen. ${ }^{19}$

Wie lauten die praktischen Anleitungen an die Leserschaft, um diese kulturelle Ausgestaltung eines „Lebenserfolgs“ für die „Gemeinschaft“20 erreichen zu können? Als erste Voraussetzung zum „Lebenserfolg“ führt C.H. Huter eine „gute Erbmasse“ an und argumentiert „eugenisch“: „Die Erbmasse ist ein wichtiger Faktor der Charakter- und Leistungsgrundlage“. ${ }^{21}$ Wer nun keine „gute Erbmasse“ besäße, hätte dank des Ratgebers andere Möglichkeiten, die „Schicksalsmächte des Erfolgs“ zu beeinflussen. Wie? Im Wissensspielraum der „NS-Rassenideologie“, die einen „rassenreinen“ Ariertypus propagierte und zugleich eine selbsterzieherische „Erhöhung und Veredelung“22 jedes „Volksgenossen“ im Rahmen der nationalsozialistischen Lebensführung in Aussicht stellte, lautet die Antwort des Glücksratgeberautors im Rückgriff auf das psycho-physiognomische Wissen seines Vaters: Wer mittels „Erziehung und Selbsterziehung eine Persönlichkeit“ herausbilde und sich entsprechend der individuellen Veranlagung beispielsweise

17 Vgl. ebd., 1.

18 Ebd., 1.

19 Zur Arbeitsideologie im Nationalsozialismus und dem Verhältnis zwischen Subjekt und „Volksgemeinschaft“ vgl. u.a. Wildt 2014b, Wildt 2018, „Arbeit macht frei“ Zughörigkeit, Ausgrenzung, Vernichtung durch Arbeit im Nationalsozialismus. In: Wildt 2019, 199-222.

20 Vgl. Huter 1940, 1.

21 Vgl. ebd., 1.

22 Hartnacke 1934, 4. 
im Gebiet der Künste, Technik oder Wissenschaft weiterentwickle, könne sich der Erfolgsmöglichkeiten sicher sein. ${ }^{23}$ Die Wissensüberlieferung der psycho-physiognomischen Methodik der „Menschkenntnis“ seines Vaters floss in die Anleitung der leistungsorientierten Optimierungspraktik des NSDAP-Mitglieds C.H. Huter ein und wurde entsprechend der NS-Arbeitsmoral umgedeutet. Auf welche Weise? „Autopädagogik“, eine „Willensschulung“ zur „Persönlichkeitskultur“, nennt der Ratgeberautor seine neu entwickelte Methodik zur „Selbsterziehung“, die ihr Wissen, nebst der Huterschen Lehre, aus der „,angewandten Psychologie“ beziehe. ${ }^{24}$ Ziel der autosuggestiven „Autopädagogik“ sei es, die in sich verborgenen „Talente zu wecken“, um einen „höheren Grad an Persönlichkeitskultur und Leistungsvermögen zu entwickeln, [...] um so ein Höchstmaß von Leistung, Erfolg und Lebensglück zu erreichen.“25

Der folgende Ratschlag zum Erlangen eines „innere[n] Glück[s]“ in Abhängigkeit zu einer leistungsorientierten Lebensführung aus dem Kapitel zur „Persönlichkeitskultur“ liest sich wie eine arbeitspsychologische und psychopolitische Synthese aus Baerwald, de Man, Giese, Arnhold und Ley:

\begin{abstract}
„Je stärker wir in unserem Beruf, unserer Rangstellung usw. sind, umsomehr [sic] haben wir die Pflichten höheren Einsatzes für die Gemeinschaft zu erfüllen. In den erfüllten Pflichten beruht auch letzten Endes unser inneres Glück, und diese stärken uns auch immer von neuem für unsere Unternehmungen und für den Weg des persönlichen Aufstieges. Wahre Persönlichkeitskultur heißt also, Selbstvertrauen gewinnen auf die Pläne und Dinge, die wir vorhaben." ${ }^{26}$
\end{abstract}

Es mag erstaunen, dass C.H. Huter zunächst ein individuell ausgerichtetes, „inneres Glück“ als Voraussetzung für den persönlichen Aufstieg als Teil einer exklusiv-individualisierten Persönlichkeitskultur beschreibt. In seiner Anweisung muss jedoch zwischen einer individuellen Berufs- und Lebensplanung, wie sie beispielsweise von Baumgarten vertreten wurde (s. Kap. 14.5), und dem hier beschriebenen leistungsorientierten Aufstieg zu einem charakterologisch-stereotypisierten „Erfolgstyp“ ${ }^{27}$ unterschieden werden. Was versteht C.H. Huter konkret unter einem „Erfolgstyp“? Wie konnten die Leserinnen erfahren, ob sie ein solcher werden könnten?

23 Vgl. Huter 1940, 1.

24 Vgl. ebd., 2.

25 Vgl. ebd., 56.

26 Ebd., 14.

27 Vgl. ebd., 17. 
Im Kapitel „Die zehn Menschentypen. Zu welchem Erfolgstyp gehören Sie?“ verknüpft C.H. Huter die „Naturell- oder Konstitutionslehre Carl Huters“ mit den „psychisch-technischen Methoden“ aus der „heutige[n] Berufs- und Begabungsforschung.“28 Das Ziel der „Erfolgs-Psychologie“ sei es, individuelle Fähigkeiten des Ratsuchenden festzustellen, „um daraus Schlüsse auf sein berufliches Vorwärtskommen“ zu ziehen..$^{29}$ Der „Erfolgstyp“ sei, laut C.H. Huter, „die Einheit verschiedener Eigenschaften und Fähigkeiten auf bestimmte berufliche und kulturelle Auswertungsmöglichkeiten im Leben“, womit der Autor für die „rassenhygienische“ Arbeitskultur sowie die leistungsorientierte Dienstbereitschaft an der „Volksgemeinschaft“ anschlussfähig wurde. ${ }^{30}$

Zur Aufschlüsselung seiner Prämissen erläutert C.H. Huter die „zehn Menschentypen“: Den „Bewegungs-Typ“, den „Wirtschafts-(Ernährungs-)-Typ“, den „Empfindungs-Typ“, das „Disharmonische Naturell“, das „Harmonische Naturell“, den „Bewegungs-Empfindungs-Typ“, den „Ernährungs-Empfindungs-Typ“, den „Bewegungs-Ernährungs-Typ“, das „Ideale-Geniale-Naturell“ sowie den „Verbrecher-Typ“. Besonders erfolgsversprechend scheint C.H. Huter die Kombination des „Bewegungs-Ernährungs-Typ[s]“ zu sein:

„Der Gesichtsausdruck zeigt mehr harte, markante Formen, welche auf Energie und sportliche Anlage, große Körperkraft, deuten. Es sind Muskel- und Tatmenschen mit zugleich wirtschaftlichen Anlagen. Solche Menschen sind impulsiv, rücksichtslos in der Durchführung ihrer Ideen und Pläne, von außerordentlicher Energie, Zähigkeit. Starker Eigenwille, Selbstbewusstsein, Ich-betontes Streben, Organisationsgabe und die Neigung, zu leiten oder zu herrschen. Vorwiegend cholerisches Temperament, begeisterungsfähig, gesellig, umsichtig, prüfend. Solche Menschen verstehen sich trotz ihrer äußeren Härte anzupassen, suchen aber leitende Positionen zu erlangen, weil sie infolge ihrer großen Energie fähig sind, industrielle und wirtschaftliche Organisationen zu leiten [...] und durch den starken Kraftausdruck andere Menschen zu erhöhter Einsatzbereitschaft anzufeuern. Das Ich-betonte Streben bezieht sich mehr auf das Erringen leitender Positionen, weniger darauf, einseitig große Güter zu erwerben [...]. Solche Menschen eignen sich für handwerkliche Berufe, als Sportlehrer, Berufssoldat, im Polizeidienst, ferner als Ingenieur und Industrieller. Wo ein solcher Mensch die Zügel in der Hand hält, wird derselbe straff, aber gerecht sein. Die Durchführungskraft ist bedeutend, die Ausdauer des Willens außerordentlich. [...]“31

Anhand einer Abbildung (Abb. 7) aus dem „Huter-Archiv“ glaubt C.H. Huter, mittels seiner psycho-physiognomischen Analyse das „Naturell“ des Erfolgstypus veranschaulichen zu können: „Der folgende Männerkopf zeigt diese Naturell-

28 Vgl. ebd., 27.

29 Vgl. ebd.

30 Vgl. ebd.

31 Huter 1940, 30 f. 
veranlagerung [sic] der harten muskulosen [sic] Gesichtsform, vortretendes Kinn, realer Blick. [...] Ein Mensch, der kraftvoll alles durchführt und rasch an das Ziel gelangt. “32

Im ersten Moment liest sich diese Beschreibung eines „Bewegungs-Ernährungs-Typ[s]“ wie das Ideal eines geschlechterstereotypisierten „Ariers“. C.H. Huters Argument für ein „Ich-betontes Streben“ widerspricht der totalitären NSIdeologie der Einheit aus Volk und Staat. In dieser Ambiguität seines Subjektivierungsdiskurses über den Erfolgstypus mag ein Grund für das zeitnahe Verbot von C.H. Huters Schriften gelegen haben, die zudem offenkundig esoterische Wissensbestände verbreiteten. Seit 1933 wurden okkultistische, esoterische und selbst „völkisch“-,,ariosophische“ Gemeinschaften neben Freimaurern, „Zeugen Jehovas" und anderen religiösen Gruppierungen im Sinne der NS-ideologischen „Gleichschaltung“ als staatsfeindliche Sekten angesehen und standen durch den „Sicherheitsdienst“ der „Schutzstaffel (SS)“ unter Beobachtung. Im Juli 1937 wurden alle sogenannten „Sekten“ verboten..$^{33}$ Gleichzeitig wurden die esoteri-

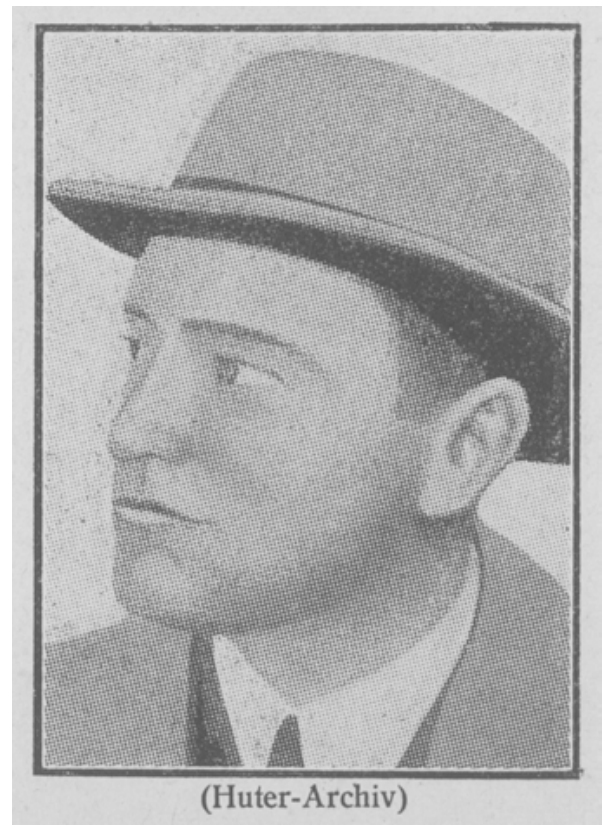

Abb. 7: C.H. Huters „Bewegungs-Ernährungs-Typ“, 1940

32 Vgl. ebd.

33 Vgl. Treitel 2004, 220 - 224. Vgl. Aerni 2012a. 
schen Interessen von NS-Elitefunktionären wie Rudolf Heß (1894-1987) und „Reichsführer SS“ (1929-1945) Heinrich Himmler (1900-1945) toleriert. Den Einfluss „völkisch“-okkulten Wissens auf die NS-Ideologie bewertet Nicholas Goodrick-Clarke als „marginal““. ${ }^{34}$ Dennoch zeigt sich eine esoterische Wissenskontinuität in nationalsozialistisch ausgerichteten Glücksratgebern über einen „arischen“35 Erfolgstypus, welche mit „völkischem“ und arbeitsmetaphysischem Wissen operierten und einen rassistisch konzipierten „Erfolgstyp“ entwarfen, wie das Beispiel C.H. Huters zeigt. Dessen esoterische Schriften konnten bis zu seiner Verhaftung 1941 verlegt werden. ${ }^{36}$

Wie lässt sich C.H. Huters Definition eines „Erfolgstyp[s]“377 wissenshistorisch im Kontext der von ihm zitierten arbeitspsychologischen „Erfolgs-Psychologie“38 im Übergang zum Nationalsozialismus verorten? Die Erkennung und Förderung von sogenannten Erfolgs- und Leistungstypen, wie sie unter anderem von Walther Moede (1888-1958), Giese und Baumgarten in ihren arbeitspsychologischen „Charakter-“ und „Eignungsprüfungen“39 entwickelt wurden, war bereits in der Weimarer Republik eine zentrale Aufgabe der psychotechnischen Betriebsopti-

34 Vgl. Goodrick-Clarke 2012a, 175.

35 Mehr zur Ariosophie, vgl. Goodrick-Clarke 2012b.

36 „Alle von meinem Vater, Carl Huter, und von mir verfaßten Werke wurden von der Gestapo vernichtet. Am 12. Juli 1941 wurde ich von der Gestapo in Dresden verhaftet und zuerst in ein Konzentrationslager Oberleutendorf bei Reichenberg in Böhmen und bald darauf in das berüchtigte Vernichtungslager Sachsenhausen bei Oranienburg/Berlin überführt. [...] Wir politischen Häftlinge erhielten ein rotes Dreieck mit einer Nummer darauf. [...] Nach der Entlassung aus dem KZ-Lager Oranienburg am 15. Januar 1942 traf ich, völlig abgemagert, in Dresden ein. Die bald darauf fällige militärische Musterung ergab einen linksseitigen Lungeninfarkt, weshalb ich a.v. geschrieben und nicht zur Wehrmacht eingezogen wurde.“ C.H. Huter 1962, 142-145.

37 Zum Erfolgstypus in der Ratgeberliteratur der Weimarer Republik vgl. Peeters 2017a, Peeters und Gruner 2018. DFG-Projekt „Poetik des Erfolgs. Institutionen und narrative Dimensionen von Erfolgsratgebern (1900 - 1933)“, Mitarbeiter: Horst Gruner und Dr. Wim Peeters, Institut für Neuere deutsche Literatur- und Medienwissenschaft, FernUniversität Hagen. Projektbeschrieb, https:// www.fernuni-hagen.de/literatur/medienaesthetik/forschung/dfg_projekt.shtml, 15.10.2020. Projektpublikationen vgl. Peeters 2015, Aschauer, Gruner und Gutmann 2015, Niehaus und Peeters 2014, Peeters 2017b, Peeters (2021).

38 Huter 1940, 4.

39 Moede war in der Weimarer Republik Vorstand des psychotechnischen Instituts der Technischen Hochschule“ in Berlin und Berater der „Deutschen Staatlichen Eisenbahnen“ gewesen und hatte nach 1924 das Amt als Vorstand des „Instituts für Wirtschaftspsychologie“ an der Handelshochschule in Berlin inne, vgl. Rabinbach 1990a, 422, Fußnote 34. Zu Moede vgl. Rabinbach 1990a, 332, vgl. Institut für industrielle Psychotechnik und Arbeitstechnik (1933-1945). In: Spur 2008, 323-409. 
mierung und Berufsberatung gewesen. ${ }^{40}$ Zudem waren sie als Teil der neusachlichen Rationalisierungsdiskurse ein wichtiger Wissensbestand vieler Glücks- und Erfolgsratgeber, wie es exemplarisch Gustav Grossmanns Ratgeber „Sich selbst rationalisieren. Wesen und Praxis der Vorbereitung persönlicher und beruflicher Erfolge“ (1927) zeigt. ${ }^{41}$

Im Kontext von C.H. Huters Rezeption der arbeitspsychologischen „ErfolgsPsychologie“ stellt sich die Frage, was nach der Machtergreifung der Nationalsozialisten mit den psychotechnischen Einrichtungen in den Betrieben und den universitären Forschungsstellen zur arbeitswissenschaftlichen Psychologie geschehen war. Im Rahmen der militärischen Aufrüstung von Hitlers „Wehrmacht“ bis zum Kriegsausbruch 1939 wurde die Eignungs- und Persönlichkeitsdiagnostik einer der bedeutendsten Arbeitsbereiche für Psycholog*innen im „Dritten Reich“.42 Selbst die Psychoanalyse wurde unter der Leitung von Matthias Göring in das „Deutsche Institut für psychologische Forschung und Psychotherapie“ integriert und staatlich gefördert. Ähnlich Gieses „Arbeitsmetaphysik“ und „Körperseelemeisterung“ war in der universitären Lehre nach einer NS-ideologischen Anpassung eine „ganzheits- und gestaltungspsychologische“ Tradition vorherrschend. ${ }^{43}$

Maik Tändler und Uffa Jensen halten in der Einleitung zu ihrem Sammelband fest, dass „psychologisches Wissen“ für die nationalsozialistische Ideologie „peripher“ blieb, weil ihr Kernelement ein biologistischer Rassismus ist. ${ }^{44}$

„Die nationalsozialistische Rassenpolitik zielte in erster Linie auf die Züchtung und Vernichtung von Körpern und die Eroberung von Räumen, weniger auf das psychologische Mikromanagement individueller Lebensführung, wie es für die Psychologisierung in liberalen, aber auch in manchen sozialistischen Gesellschaftssystemen charakteristisch war und ist. “45

In den folgenden Kapiteln soll der Versuch unternommen werden, den peripheren Status von psychologischem Wissen in der NS-Arbeitsideologie zu hinterfragen, indem das „psychologische Mikromanagement individueller Lebensführung“

40 Eine Auswahl: Vgl. Baumgarten 1927b, Baumgarten 1930, Baumgarten 1941, Baumgarten 1942, Baumgarten-Tramer 1945, Giese 1927, Giese 1928a, Giese 1928b, Moede 1930a, Moede 1930b, Giese 1931a, Giese 1934.

41 Vgl. Grossmann 1927. Ferner vgl. Kleiner und Suter 2015.

42 Vgl. Tändler und Jensen 2012, 27, Killen 2007, 68.

43 Mehr zur Ganzheits- und Gestalttheorie der angewandten Psychologie im Nationalsozialismus vgl. Prinz 1985, Métraux 1985, 245-248, Rabinbach 1990b, 282-8, Gessner 2019.

44 Vgl. Tändler und Jensen 2012, 28.

45 Ebd. 
exemplarisch anhand der Untersuchung von Glückswissen in der NS-Arbeitspsychologie und der massenmedialen Ratgeberliteratur als ein Teil einer wissenshistorischen Alltagskultur in den Blick genommen wird. Dabei wird die These aufgestellt, dass es nach 1933 - trotz radikaler ideologischer Brüche - auch wissenshistorische Kontinuitäten in der arbeitspsychologischen Psychotechnikforschung in Bezug auf eine spezifisch affirmative Gefühlspolitik gab: Ein „Arbeitsfreude“ generierendes Kultur- und Selbstbewusstsein (Münsterberg/de Man) wurde „rassenhygienisch“ zur Kultur einer „Arbeitsfreude“ im Dienst der „Volksgemeinschaft“ (Ley) umgedeutet. Daran anschließend stellt sich die Frage: Welche Bedeutung kam der arbeitspsychologischen Forschung zum ,arischen“ Erfolgstypus im Kontext der „rassenhygienischen“ NS-Arbeitswissenschaft zu?

\subsection{Arbeitspsychologische Forschung zum „arischen“ Erfolgstypus im Nationalsozialismus}

Die Förderung von charakterologischen „Leistungstypen“ im Betrieb erhielt im Nationalsozialismus im Rahmen der autoritären „Führungsideologie“46 eine neue Bedeutung. Zahlreiche arbeitspsychologische Forschungen dokumentieren eine „rassenhygienische“ Leistungsauslese bzw. „Menschenauslese““.47 Exemplarisch seien hier die „völkischen“ Forschungsarbeiten von Erich Grassl, „Die Willensschwäche. Gleichzeitig ein Beitrag zur Theorie des Willens, der Willensentwicklung und Willenserziehung“ (1937), Ehrig Wartegg, „Gestaltung und Charakter. Ausdruckdeutung zeichnerischer Gestaltung und Entwurf einer charakterologischen Typologie“ (1939) sowie Heinz Remplein, „Beiträge zur Typologie und Symptomatologie der Arbeitskurve“ (1942), zu nennen. ${ }^{48}$

Das „arische“ Leistungsprinzip galt, im Sinne der totalitären NS-Arbeitsmoral, auch für Wissenschaftler^innen, welche zur Arbeitspsychologie forschten, wie das folgende Beispiel zeigt. Im Juni 1933 publizierten die Arbeitspsychologen Walther Moede (1888-1958), „Reichsbahnrat“ und Psychotechniker Richard Couvé sowie der Oberingenieur und Psychotechniker K[arl] A[ugust] Tramm den „Aufruf der Gesellschaft für Psychotechnik“ in der Fachzeitschrift „Industrielle

46 Vgl. Briefs 1934, $117 \mathrm{f}$.

47 Zur ,völkischen Leistungsgemeinschaft“ im Nationalsozialismus, vgl. Konkurrenz und Kooperation - Die wissenschaftliche Landschaft während des „Dritten Reichs“. In: Hachtmann 2012, 301-311. Vgl. Lohn und Leistung: Leistungsbezogene Lohnsysteme und Arbeiterbewertungsverfahren. In: Hachtmann 1989, 161-223, insb. $161 \mathrm{ff}$.

48 Vgl. Grassl 1937, Wartegg 1939, Remplein 1942. 
Psychotechnik““. ${ }^{49}$ Die Psychotechniker*innen sollten es unter dem NS-propagandistischen Schlagwort „Gemeinnutz vor Eigennutz“ als eine „Pflicht zur Mitarbeit am Staat empfinden“, der "gleichgeschalteten“ Gesellschaft per 1. August 1933 beizutreten. ${ }^{50}$

Bereits der erste „Kongreß der Deutschen Gesellschaft für Psychologie“, der vom 16. bis 19. Oktober 1933 in Leipzig stattfand, machte in der Eröffnungsrede des Sächsischen „Ministers für Volksbildung“, Dr. Wilhelm Hartnacke (1878-1952), die zukünftige Zusammenarbeit der arbeitspsychologischen Forschung im totalitären „Dienst“ des „Führer[s] Adolf Hitler“51 und der „Volksgemeinschaft“ deutlich:

„Was fordert und fördert der neue Staat als Wissenschaftsbetrieb, und was lehnt er ab? Die Wissenschaft ist in die Ganzheit unseres Seins einbezogen, und sie darf sich an keiner Stelle mit dem Wohle des Ganzheitsstaates stoßen oder gar feindlich berühren. Wissenschaft, die sich den Interessen des Ganzheitsstaates entgegenstellt, ist als volksfeindlich abzulehnen. [...] Das Wissen, das er [der neue Staat] zu pflegen und zu fördern sucht, muß der Erhöhung und Veredelung des inneren Seins dienen, es muß letztlich irgendwie bezogen sein auf Volk und Staat und Volk und Staat dienen." ${ }^{\text {"52 }}$

Das NS-Regime verlange, laut Hartnacke, eine „gleichgeschaltete“ Wissenschaft, die eine NS-ideologisch konforme Wissensproduktion garantiere und keine wissenschaftliche Diskursivität und „volksfeindlich[es]“ Gedankengut toleriere. Welche Forschungsfelder prioritär zu behandeln seien und welche Bedeutung die Psychologie in diesem „gleichgeschalteten“ Wissenschaftsszenario hatte, wird im folgenden Ausschnitt der Rede deutlich:

„Am höchsten steht, was bezogen ist auf Bestandessicherung und Hebung des Volkes, auf Sicherung seines Daseins und Erhaltung des naturgegebenen Rassenerbgutes und der besten geistigen Form [...] volksbiologisches Denken muß als Erziehungsziel durchgeführt werden [...] Daher kommt Wissenschaft im Dienste der Erbgutpflege vor Bildungspflege. [...] Die Psychologie als Wissenschaft von den Vorgängen im Innenleben ist die Wissenschaft, die an die allertiefsten Fragen des persönlichen und vor allem des völkischen Seins rührt, an die

49 Zu Moede vgl. Spur 2008. Eine Werkauswahl: Vgl. Moede 1920a, Moede 1930a, Einleitung zu „Psychologie der Masse“ von Gustav Le Bon (5., dt. Aufl. 1932), vgl. Le Bon 1932, Moede 1935, Moede 1943. Zu Couvé vgl. Couvé 1925, Couvé 1934. Zu Tramm vgl. Tramm 1921, Tramm und Heesemann 1935.

50 Vgl. Moede und Couvé 1933.

51 Vgl. Hartnacke 1934, 5.

52 Ebd., 4. 
Fragen nach den Möglichkeiten und Mitteln der Formung des geistigen Menschen. Können wir den Menschen nach Belieben formen, wie wir ihn brauchen für Volk und Staat?“53

Hartnacke verknüpfte sein „volksbiologisches Denken“ mit dem leistungsorientierten Auftrag an die Psychologie, „Mittel“ zur „Formung“ der menschlichen Psyche zu erarbeiten und damit als „Dienerin und Führerin“ von „Erziehungsfragen“ von der „NS-Rassenideologie“ instrumentalisiert zu werden. ${ }^{54}$ Die erarbeiteten psychologischen Methoden sollten ausschließlich im Interesse des totalitären Einheitsstaates aus „Volk und Staat“ entstehen. So lautete die Botschaft an die anwesenden Wissenschaftler`innen im Saal, die sich fortan dem „rassenhygienischen“ Forschungsinteresse zur utopischen „Erbgutsicherung“ widmen sollten oder, alternativ, zu Staatsfeinden wurden:

„Daß das Wahre an Erbgutsicherung sich verbinde mit den wertvollsten psychologischen Erkenntnissen für die Volkserziehung, das ist mein Wunsch für die deutsche Zukunft. “55

Hartnacke definierte den Sinn und Zweck der zukünftigen psychologischen Forschung als „Dienst“ am „Führer Adolf Hitler, diese[m] große[n] Psychologen“. ${ }^{56}$

Vergleichbar mit den nationalsozialistischen Ratgeberautor*innen appellierte der Redner (ähnlich den Worten C.H. Huters) an die selbstdisziplinarische „Willensschulung“ und „Selbsterziehung“ seiner Hörer^innen. Totalitären „Pflicht- und Opfersinn und Liebe“ forderte der Ministerpräsident von den ,Menschenkenner*innen', um die rassistische Utopie eines „Erbgutes zu edlem Menschentum“ und „segenvolle[m] Wirken im neuen Staate und für das deutsche Volk“ erforschen und lehren zu können: „In diesem Sinne Sieg Heil dem neuen deutschen Willen zu Leben und Wirken in Arbeit, Zucht und Ehre. “57

Wie wurde dieser Appell an die Psycholog*innen im Kontext der nationalsozialistischen Arbeitsmoral „Kraft durch Freude“ - in der Vorstellung des „neuen

\section{Ebd.}

54 Vgl. ebd., 5.

55 Ebd., 5.

56 Vgl. Hartnacke 1934, 5.

57 Ganze Passage: „Möge diese erste Tagung der deutschen Psychologen im neuen Reiche unseres Führers Adolf Hitler, dieses großen Psychologen aus innerer Schau heraus, einen Grundstein und Ausgangspunkt bilden zu segenvollem Wirken im neuen Staate und für das deutsche Volk. Denn ihm, seinem ewigen Leben, dienen wir alle. Es wird leben, wenn es sich ständig aus seinem besten Blute und Erbgute erneuert und wenn Wissenschaft und Bildung und Volkserziehung mit Pflicht- und Opfersinn und Liebe daran arbeiten, daß die geprägte Form des Erbgutes zu edlem Menschentum in Volksgemeinschaft sich entwickelt. In diesem Sinne Sieg Heil dem neuen deutschen Willen zu Leben und Wirken in Arbeit, Zucht und Ehre.“ Hartnacke 1934, 5. 
deutschen Wille[ns] zu Leben und Wirken in Arbeit, Zucht und Ehre“ - mit Blick auf C.H. Huters Konzept eines charakterologischen Erfolgstypus in der Arbeitswissenschaft umgesetzt? Zur wissenshistorischen Kontextualisierung dienen exemplarische Beispiele stark rezensierter Arbeitspsycholog*innen, Arbeitsmetaphysiker^innen und Betriebssoziolog^innen wie Goetz Briefs, Ludwig Klages und Walter Malmsten Schering.

Der Wirtschaftssoziologe Goetz Briefs (1889-1974) beobachtete seit der nationalsozialistischen „Gleichschaltung“ eine „militärische Führungsideologie“ in den Betrieben. Diese ,neue“ Arbeitsideologie stand dem Verfasser von Schriften wie „Untergang des Abendlandes. Christentum und Sozialismus: Eine Auseinandersetzung mit Oswald Spengler“ (1920) zufolge in wissenshistorischer Kontinuität zum „[m]ilitärisch-[d]isziplinäre[n]“ Führungsstil der Preußischen Armee. ${ }^{58}$ In der „neue[n] gesellschaftliche[n] Vorformung des Betriebs“ sah Briefs eine Vorschau der bevorstehenden gesellschaftlichen Entwicklungen des sich im Aufbau befindenden totalitären NS-Regimes, wie sie schon vom „völkisch“-nationalistischen Publizisten Arthur Moeller van den Bruck (1876-1925) in „Der Preußische Stil“ (1912) aufgegriffen worden war. ${ }^{59}$

Beim metaphysischen Charakterologen und Graphologen Ludwig Klages (1872-1956) oder beim Psychologen und Soziologen Walter Malmsten Schering (1883-1954) kam eine biologistische und (para-)militärische „Führerqualität“ als „vital-ethische Einheit“ zur Sprache, die sich angeblich reibungslos von einer NS-„,rassenmoralischen“ Betriebsführung der „deutschen Arbeiter“ auf die Führung der „Wehrmachtsoldaten“ weiterdenken ließ: „In der Sinngebung liegt die Wurzel des Erfolgs des charaktervollen Menschen, seine Überzeugungskraft und damit seine Führerqualität [...]. Der Charakter liegt daher in dem, was im Kampfe sich bewährt, was also nicht bloß kampffähig ist, sondern auch den Kampf aufnimmt."60

Auch der Arbeitspsychologe und Ratgeberautor Giese folgte dem Ruf der „Psychotechnischen Gesellschaft“. In seiner mehr „kulturphilosophischen“61 als arbeitswissenschaftlichen Schrift „Nietzsche - Die Erfüllung“ (1934), propagierte Giese die ,neue“, „arische“ „Körperkultur“. Eine Einheit aus „Körper“, „Geist“ und „Seele“ sieht Giese im männlichen Ariertypus versinnbildlicht. Mit dem Zitat aus Nietzsches „Zarathustra“: „Könnt Ihr einen Gott schaffen? So schweigt mir doch von allen Göttern. Wohl aber könntet Ihr den Übermenschen schaffen“, glaubt

58 Vgl. Briefs 1934, $122 \mathrm{f}$.

59 Vgl. Ebd. Vgl. Moeller van den Bruck 1916.

60 Schering 1937, 106f. Zur Charakterologie und Graphologie bei Klages vgl. Klages 1936, Klages 1941. Ferner vgl. Giese 1934.

61 Giese 1934, 191. 
Giese, den Beweis für die wissenshistorische Kontinuität zu Hitlers NietzscheRezeption erbringen zu können. ${ }^{62}$ Als Beispiel für die „Übermensch“-Rezeption Hitlers dienen Giese außerdem Passagen aus „Mein Kampf“ sowie dessen Rede am „Deutschen Turnfest“ 1933. Die nationalistischen Erziehungskonzepte des Sportpädagogen Friedrich Ludwig Jahn (1778-1852) hätten Hitler als personifiziertes Vorbild für einen „arischen“ und leistungsorientierten Menschentyp gedient. ${ }^{63}$ „Der Zarathustra-Übermensch wird Symbol für den erstrebenswerten Typ“, so Giese. ${ }^{64}$

Was in den kommenden Jahren vom Nationalsozialismus zu erwarten sei, skizziert Giese in der Folge mit Hilfe von „eugenischen“ und „rassenhygienischen“ Konzepten. Sein Literaturverzeichnis liest sich wie ein ,Who is Who“ der antisemitischen „Rassenideologen“ und „Kulturphilosophen“ jener Zeit. Giese nimmt beispielweise Bezug auf Ludwig Klages’ „Der Geist der Widersacher der Seele“ (1929), Martin Heideggers „Was ist Metaphysik?“ (1930), Ernst Günther Gründels „Jahre der Überwindung“ (1934), Alfred Rosenbergs „Der Mythos des 20. Jahrhunderts“ (1933), Oswald Spenglers „Untergang des Abendlandes, Bd. 2“ (1922), Moeller van den Brucks „Das Dritte Reich“ (1931) sowie „Das ewige Reich, Bd. 2“ (1934) und Alfred Baeumlers „Männerbund und Wissenschaft“ (1934). Er scheut sich auch nicht, das Sonderheft der „Nationalsozialistischen Monatshefte“ (1934) zum Thema „Zarathustra“ zur ideologischen Unterstreichung seiner „Übermensch“-Rezeption zu zitieren. ${ }^{65}$

Anhand der Lektüre von „Nietzsche - Die Erfüllung“ wird der ideologische Wandel Gieses deutlich. ${ }^{66}$ Dieser Wandel vollzog sich seit spätestens den 1920er Jahren, ausgehend von Gieses Rezeption einer amerikanischen Körperkultur als eine kulturnationalistische ,Glückskultur begriffen. Giese konstruierte für Deutschland einen körperpolitischen Entwicklungsprozess: von einem weiblich stereotypisierten Amazonentypus als kriegerischem Massenkörper bis hin zur „rassenhygienischen“ Körperkultur eines „nordischen Menschen“. In seinem Konstrukt einer patriarchalen Gesellschaftsordnung entwarf der Arbeitspsycho-

62 Vgl. ebd., 57.

63 Vgl. Giese 1934, 56f. Zur wissenshistorischen Bedeutung des Sports im Nationalismus vgl. Czech 1994, Williams 2007, Bahro 2013, Becker und Schäfer 2016, insb. Timpe 2016, Hachtmann 2016. Mehr zur Turnerbewegung s. Kap. 16.5.

64 Vgl. Giese 1934, 57.

65 Von Giese zitierte Ausgaben: Klages 1929, Heidegger 1930, Gründel 1934, Rosenberg 1933, Spengler 1922, Bruck 1931, Moeller van den Bruck 1934, Baeumler 1934, Nationalsozialistische Deutsche Arbeiterpartei 1934.

66 Vgl. Killen 2007, 69. 
loge an der Spitze den männlichen „Arier“ bzw. den nationalsozialistischen Führungstypus als leistungsorientierten „Erfolgstyp“ ${ }^{67}$ Diese Analogie zwischen einem anzustrebenden charakterologischen Führungstypus und einem leistungsorientierten Erfolgstypus zirkulierte als wissenspraktische Anleitung auch in der Ratgeberliteratur mit Blick auf einen NS-Arbeitersoldatentypus, wie im nächsten Kapitel 12.3 aufgezeigt wird. Gefragt wird: Welche praktischen Anwendungsbereiche fand das arbeitspsychologische Glückswissen betreffend einem leistungsorientierten Erfolgstypus im Kontext des Zweiten Weltkriegs?

\section{3 „Der Weg ins Glück“: Vom leistungsorientierten Führungstypus zum „opferbereiten“ Arbeitersoldaten im Zweiten Weltkrieg}

Der Ratgeber „Der Weg ins Glück. Durch Selbstbemeisterung zur Lebensmeisterschaft“ (1941) von Will Rinne zeigt exemplarisch, wie Wissen der arbeitspsychologischen Charakterologie über einen angeblichen Erfolgstypus für das Selbstmanagement einer individuellen Lebensführung im Alltag und zugleich in der militärischen „Kriegsführung“ eines „Wehrmachtsoldaten“ nutzbar gemacht werden konnte. Das Psychowissen über einen charakterologischen Führungstypus diente dem Autor nicht nur zur Erfolgsanleitung für eine „glückliche“ Lebensführung und berufliche Karriereplanung, sondern auch übergangslos für die zielgerichtete militärische Laufbahn eines „opferbereiten“ Kriegssoldaten (wie dies auch die nationalsozialistische „Führungslehre“ von Hans Bernhard Brausse dokumentiert). ${ }^{68}$ Wie dieser Wissenstransfer erfolgte, zeigt die folgende Analyse.

Der Ratgeberautor und Verlagsdirektor von Schriften wie „Unsterbliches

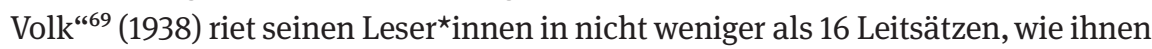
(ähnlich dem Ansatz C.H. Huters) der Weg von der „Selbstbemeisterung zur Lebensmeisterschaft“ gelingen könnte: „Erkenne dich“, „Fasse Mut“, „Sei froh“, „Lerne sehen“, „Lausche“, „Schweige“, „Vergeude keine Kraft“, „Sage nein“, „Liebe die Wahrheit“, „Lerne atmen“, „Entspanne dich“, „Nutze jede Geisteskraft“, „Pflege dein Gedächtnis“, „Sei selbständig“, „Konzentriere dich“ und

67 Zur dichotonen Darstellung von Geschlechterstereotypen und Körperkultur im Nationalsozialismus vgl. Wildmann 1998, Diehl 2006, Schmidtke 2007, Frietsch und Herkommer 2009, Frevert 2010, Beck u. a. 2016.

68 Vgl. „Die Kunst der Führung“, Brausse 1937. Vgl. auch Brausse 1940.

69 Rinne 1938. 
„Wolle“ ${ }^{70}$ „Leb mit der Welt in Frieden - Schon winkt die Burg - Am Ziel“. So lauteten die drei pseudo-pazifistischen Schlusskapitel seines Ratgebers.

Rinne postuliert ein „geistige[s] Glück der Erkenntnis“ mithilfe unterschiedlicher Anleitungen zu einer zielgerichteten Lebensführung entsprechend den eigenen Fähigkeiten und Charaktereigenschaften und ergänzt diese mit „Körpergymnastik“, Atemübungen, Konzentrationsübungen, Ernährungsberatung und sozialen Verhaltensregeln. Was verstand der Autor unter einem ,geistige[n] Glück der Erkenntnis“? Dieses „Glück“ läge in der „Erkenntnis“, dass „Selbstveredelung durch das beseelte Glück der Dienstbereitschaft und des Dienens zum durchstrahlten Glück [...]“ hindurchdringen könne. ${ }^{71}$

In einem autoritären Ton tadelt Rinne zu Beginn des Ratgebers seine Leser*innen für ihre charakterlichen, psychischen wie auch physischen Unzulänglichkeiten und kündigt seinem Leserpublikum eine heilsbringende Botschaft in Form einer autosuggestiven Selbstdisziplinierungspraktik an: „Du zweifelst? Du kannst das nicht? Du kannst es, und du wirst dein Ziel erreichen. Ich zeige dir den Weg, mit deiner Kraft dich selber zu meistern." ${ }^{\text {"72 }}$ Als roter Faden zieht sich in Rinnes Leitsätzen die Idee einer autosuggestiven „Selbsterziehung“ zum „Helden“, in Referenz an die griechischen Heldenepen, durch. ${ }^{73}$ Der stereotypisierte männliche Heros aus der griechischen Mythologie erfährt bei Rinne, unter Ausblendung antiker Heroinnen, eine einseitige, geschlechterspezifische Umdeutung. Im Zweiten Weltkrieg sollte das „rassenhygienische“, ideologisch überhöhte Ziel der „Selbstzucht“-Praktik die „Selbstveredelung“ in der Soldaten- und „Führer“Werdung verkörpern.

Die Machtgewinnung über die Mitmenschen war nach Rinne, wie schon bei C.H. Huter, eine zentrale Eigenschaft des anzustrebenden Führungstypus. Dies zeigt sich im Kapitel zur Konzentrationsübung in der Tradition der antiken (männlichen) Philosophen und rhetorisch begabten Juristen und Politiker: „Wenn du diese Selbstzucht der Gedanken durch die Kraft der Konzentration gewonnen hast, dann bist du deiner Umwelt überlegen. Du wirkst dich als Macht aus, zwingst in deinen Bann und siegst." ${ }^{\text {"74 }}$ Dieser scheinbar lineare Entwicklungsprozess von der individualpsychologischen Wirkungsmacht des leistungsorientierten Erfolgstypus im (Berufs-)alltag zur massenpsychologischen Wirkungsmacht des charakterologischen Führungstypus im Krieg war eine Wissenskontinuität, die aus der arbeitspsychologischen Forschung zur „Men-

70 Vgl. Rinne 1941, 2.

71 Vgl. ebd., 48.

72 Vgl. ebd., 4.

73 Vgl. ebd. Zur Rezeption der Antike im Nationalsozialismus vgl. Chapoutot 2014.

74 Rinne 1941, 37. 
schenführung“ und den Ratgeberpraktiken der Weimarer Republik in die NS-Arbeitsmoral „Kraft durch Freude“ transferiert wurde. Dies wurde bereits bei Giese und C.H. Huter gezeigt. Als Beispiel für einen Ratgeber, der diese Wissenspraktiken bereits 1931 verband, sei Adolf Friedrichs Schrift „Über Menschenführung. Der wahre Führer wirkt nicht durch krampfhafte Willensanstrengung, sondern durch kraftvolles Ausstrahlen der Persönlichkeit“" genannt. ${ }^{75}$

Im Kapitel „Sei froh“ verschränkt Rinne die NS-Arbeitsideologie „Kraft durch Freude“ mit den arbeitspsychologischen Metaphern von „Rhythmus“ und „Arbeit“, wie gesehen bei Gieses Untersuchung der „Girlkultur“. Rinne beschreibt dieses Gefühlserlebnis aus der wissenshistorischen Tradition esoterischer Energie- und post-nietzscheanischer „Willenskraft“-Lehren heraus in Form eines Versprechens auf ein musikästhetisch vergleichbares, körperpolitisches Glückserlebnis:

\footnotetext{
„Das Leben ist Mannigfaltigkeit und Fülle, in seinem Schwung und seiner Kraft, in seinem unbändigen Rhythmus und seiner jauchzenden Freude [...] Und an jedem neuen Tag sprich beim Erwachen: ,Ich werde heute froh sein!‘ Sag’s zehn-, sag’s zwanzigmal mit der Inbrunst eines Gebets und mit dem Wissen der Erfüllung. [...] Damit transportierst du dein Leben aus trübem Moll in strahlendes Dur." ${ }^{\text {"76 }}$
}

In den Schlusskapiteln relativiert der Ratgeberautor Rinne, wie schon Baerwald und C.H. Huter, die menschliche „Glücksmöglichkeit“"77: „Doch dieses geistige Glück der Selbsterziehung, der Selbstverantwortung und der Selbstveredelung ist nicht unser Glück.“78 Wessen „Glück“ sei es dann? Die Antwort sollte, angesichts der Analysebeispiele zur Politik der ,Glückskulturen‘, nicht länger überraschen: „Das beseelte Glück der Kameradschaft, das beseelte Glück der Dienstbereitschaft und des Dieners ist jedes Opfers wert. “79 Die Arbeit an der eigenen „Persönlichkeit“ sei zentral, um das „Glück der Dienstbereitschaft“ zu erlangen, wie schon bei C.H. Huter gesehen. Mit dieser Polemik einer autosuggestiven und selbstdisziplinarischen Persönlichkeitsschulung, mit Verzicht auf individuelles „Glück“ und ein langes, friedvolles Leben fernab des Krieges, glaubte der Ratgeberautor Rinne die vorherigen Leitsätze legitimieren zu können.

Im Schlusskapitel ergänzt er seine Ratschläge mit der moralischen Frage nach einem ,guten‘ Leben. Indem Rinne die Tugendhaftigkeit eines Menschen hervor-

75 Für diesen Hinweis danke ich Wim Peeters.

76 Rinne 1941, $7 \mathrm{f}$.

77 Vgl. ebd., 41.

78 Vgl. ebd., 43.

79 Vgl. ebd., 45. 
hebt, die er in der ,Rolle“ des „Helfer[s]“ und „Führer[s]“ anderer „Volksgenossen“ definiert, glaubte er, die „rassenmoralische“ „Opferbereitschaft“ für die „Volksgemeinschaft“ im aktuellen Kriegsgeschehen rechtfertigen zu können. Diese „Ritterpflicht“ als „höchste[...] Stufe des Glücks“ preist Rinne in überschwänglichen, romantizistischen Naturmetaphern, welche die gegenwärtige Kriegskatastrophe symbolistisch verklärten. ${ }^{80}$

Anhand Rinnes Ratgeber ließ sich exemplarisch aufzeigen, wie arbeitspsychologische und esoterische Wissensdiskurse über „Wille“ und „Kraft“ zur „Selbstveredelung“ im Nationalsozialismus einen Wandel erfuhren. Als kollektives „Glück“ „der Dienstbereitschaft“81 an der „Volksgemeinschaft“ wurden diese Wissensbestände für den deutschen Arbeitertypus seit den Aufbaujahren 1933-1939 „rassenideologisch“ umgedeutet. In der Kriegspropaganda des Zweiten Weltkriegs wurde ein kollektivistisches „Glück der Dienstbereitschaft“ für den Soldatentypus als „opferbereite“ Durchhalteparole zur militärstrategischen und selbstdisziplinarischen „Selbstbemeisterung“ instrumentalisiert.

Abschließend seien als Beispiel für einen praktischen Anwendungsbereich der „Führungs-Psychologie“ die Abhandlungen zur „Wehrpsychologie“ genannt, die vom „Psychologischen Laboratorium des Reichskriegsministeriums“ in Berlin herausgegeben wurden. ${ }^{82}$ Im Vorwort des Sammelbandes erläutern Oberregierungsrat und Leiter der „Wehrpsychologie“ Max Simoneit (1896-1962) ${ }^{83}$ und Oberst Hans von Voß (1875-1966), 1939-1942 Leiter des Laboratoriums, das Anwendungsfeld der „Wehrpsychologie“, indem sie den theoretischen und methodischen Wissenstransfer aus der Arbeitspsychologie offenlegen:

„Der Aufbau der Wehrmacht hat auch erneuten Aufschwung der Wehrmachtpsychologie gebracht. [...] Soziologische Psychologie der soldatischen Arbeit und der Arbeitsmittel, charakterologische Psychologie der Menschenauslese, Psychologie der Ausbildung und Erziehung, Psychologie der Propaganda und Völkerpsychologie, Psychologie des Kampfes und der Kriegsführung gehören als Einzeldisziplinen zum System der Wehrmachtpsychologie." 84

Die anschließende Verteidigung der empirischen Evidenz der psychotechnischen „Wehrpsychologie“ im Vergleich zur gescheiterten psychotechnischen Arbeits-

80 Vgl. ebd., 46. Zur Rezeption der deutschen Romantik im Nationalsozialismus vgl. Klausnitzer 1999.

81 Vgl. ebd., 48.

82 Vgl. Psychologisches Laboratorium des Reichskriegsministeriums Berlin 1936. Zur „Wehrmachtpsychologie“ vgl. Flik 1989, Wolfradt, Billmann-Mahecha und Stock 2017.

83 Zu seinen Werken vgl. u.a. Simoneit 1933, Simoneit 1943.

84 Voß und Simoneit 1936, 3 [Werbebroschüre zur Publikation]. 
psychologie in den Betrieben der Weimarer Republik ist von wissenshistorischer Relevanz. ${ }^{85}$ Ähnlich ihren Vorgänger*innen sahen sich auch die Wehrmachtpsycholog^innen zunehmend in einem wissenschaftlichen Legitimationsstreit, den sie schlussendlich verloren. 1942 wurde die „Wehrmachtspsychologie“, die sich vor allem auf die psychotechnische Auswahl, Beratung und das Training von Offizieren konzentrierte, offiziell aufgelöst. ${ }^{86}$

\subsection{Fazit}

Das interdisziplinäre Glückswissen der Arbeitspsychologie, Betriebssoziologie, Charakterologie und metaphysischen Physiognomik über einen charakterologischen Erfolgstypus aus der Zeit der Weimarer Republik erfuhr im Übergang zum totalitären NS-Regime eine arbeitspsychologische Umdeutung. Das nationalsozialistische, autoritäre und rassistische „Führerprinzip“87 kannte unterschiedliche Anwendungsbereiche. In den verschiedenen NS-Parteiorganisationen reichte dies von der Bestimmung geeigneter „Gauleiter“, „Bund-deutscher-Mädel“-Führerinnen oder „Hitler-Jugend“-Führer bis hin zur Ernennung von Funktionären der „Waffen-SS“ und Offizieren der „Wehrmacht““ ${ }^{88}$ Der Jurist Hans Bernhard Brausse (1908 - 1977) verfasste beispielsweise 1940 „Die Führungsordnung des deutschen Volkes. Grundlagen einer Führungslehre“ für die „arischen“ „Volksgenossen“89.

Im folgenden Kapitel 13 soll aus einer geschlechterhistorischen Perspektive der arbeitspsychologische Anwendungsbereich eines stereotypisierten Erfolgstypus anhand der autobiografischen Erfahrungsberichte junger Menschen aus dem „NS-Arbeitsdienst“ untersucht werden.

85 Ebd.

86 Vgl. Flik 1989.

87 Zum Begriff vgl. u.a. Kershaw 1998.

88 Zur Doppelrolle des Soldaten und „Volksgenossen“ vgl. Frevert 2001. Zu Frauen im NS-Regime vgl. Frevert 1989, Steinbacher 2007, Kramer 2011.

89 Vgl. Brausse 1940. Vgl. auch Brausse 1937. 